\section{Activin receptor patterning of foregut organogenesis}

\author{
Seung K. Kim, 1,7,8 Matthias Hebrok, ${ }^{2,6,7} \mathrm{En} \mathrm{Li}^{3}{ }^{3}$ \\ S. Paul Oh, ${ }^{4}$ Heinrich Schrewe, ${ }^{5}$ Erin B. Harmon, ${ }^{1}$ \\ Joon S. Lee, ${ }^{1}$ and Douglas A. Melton ${ }^{2,8}$ \\ ${ }^{1}$ Department of Developmental Biology, Stanford University, \\ Stanford, California 94305-5329 USA; ${ }^{2}$ Howard Hughes \\ Medical Institute and Department of Molecular and Cellular \\ Biology, Harvard University, Cambridge, Massachusetts 02138 \\ USA; ${ }^{3}$ Cardiovascular Research Center, Massachusetts \\ General Hospital, Charlestown, Massachusetts 02129 USA; \\ ${ }^{4}$ Department of Physiology, University of Florida, Gainesville, \\ Florida 32610 USA; ${ }^{5}$ Department of Developmental Biology, \\ Max-Planck-Institut für Immunbiologie, 79108 Freiburg, \\ Germany; ${ }^{6}$ Diabetes Research Center, Department of \\ Medicine, University of California, San Francisco, California \\ 94143-0573 USA
}

Foregut development produces a characteristic sequence of gastrointestinal and respiratory organs, but the signaling pathways that ensure this developmental order remain largely unknown. Here, mutations of activin receptors ActRIIA and ActRIIB are shown to disrupt the development of posterior foregut-derived organs, including the stomach, pancreas, and spleen. Foregut expression of genes including Shh and IsII is shifted in mutant mice. The endocrine pancreas is particularly sensitive to the type and extent of receptor inactivation. ActRIIA ${ }^{+/-} \mathrm{B}^{+/-}$ animals lack axial defects, but have hypoplastic pancreatic islets, hypoinsulinemia, and impaired glucose tolerance. Thus, activin receptor-mediated signaling regulates axial patterning, cell differentiation, and function of foregut-derived organs.

Received March 28, 2000; revised version accepted May 15, 2000.

Organs, including the lungs, stomach, spleen and pancreas, develop at stereotyped positions along the anterior-posterior (AP) axis of the vertebrate foregut. Signaling pathways that govern skeletal and central nervous system AP patterning have been identified (Sasai and De Robertis 1997; McPherron et al. 1999), but comparatively little is known about the genetic programs that specify foregut organ position, morphogenesis, and cellular differentiation. Restricted endodermal expression of Sonic hedgehog (Shh), a member of the Hedgehog family of secreted signaling proteins, specifies domains within the gut to generate boundaries and proper differentiation of the stomach, spleen, pancreas, intestines,

[Key Words: Endoderm; pancreas; activin; foregut; diabetes mellitus; Hedgehog]

${ }^{7}$ These authors contributed equally to this work.

${ }^{8}$ Corresponding authors.

E-MAIL seungkim@cmgm.stanford.edu; FAX (650) 725-7739.

E-MAIL dmelton@biohp.harvard.edu; FAX (617) 495-8557. and respiratory tract (Roberts et al. 1995; Apelqvist et al. 1997; Kim et al. 1997a,b; Hebrok et al. 1998; Kim and Melton 1998). Shh misexpression or inactivation in mice leads to defects in foregut organogenesis that mimic common human congenital disorders, including tracheal-esophageal fistula, heterotopic pancreas, annular pancreas, and hyposplenism (Apelqvist et al. 1997; Hebrok et al. 1998, 2000; Kim and Melton 1998; Litingtung et al. 1998; Pepicelli et al. 1998).

Activins, members of the transforming growth factor- $\beta$ (TGF- $\beta$ ) superfamily, govern embryonic axial patterning (Hoodless et al. 1999), and may restrict Shh expression in embryonic chicks (Levin et al. 1995; Hebrok et al. 1998). Type II activin receptors, ActRIIA and ActRIIB, are cell-surface protein kinases, and previous work has demonstrated that ActRIIB-mediated signaling controls both AP pattern of the axial skeleton and lateral asymmetry of heart and lungs (Oh and Li 1997). Following gastrulation, and during organogenesis in mid-gestational embryos, both ActRIIA and ActRIIB are expressed in the primordia of foregut organs, including lungs, stomach, intestines, and pancreas (Feijen et al. 1994; Manova et al. 1995; Verschueren et al. 1995). In adult mice, both ActRIIA and ActRIIB are expressed in pancreatic islet cells (Yamaoka et al. 1998; Shiozaki et al. 1999). Thus, ActRIIA and ActRIIB are transcribed in foregut organ anlagen before and during morphogenesis and cellular differentiation. To study type II activin receptor function in foregut patterning, we examined embryos with combinations of ActRIIA and ActRIIB null mutations derived by gene targeting (Oh and Li 1997; Song et al. 1999). In this study we show that type II activin receptors are crucial for normal development of many foregut organs, and that these receptors have a particularly critical role in development and function of the endocrine pancreas.

\section{Results and Discussion}

ActRIIA $^{-/-} \mathrm{B}^{-/-}$and ActRIIA ${ }^{-/-} \mathrm{B}^{+/-}$embryos failed to gastrulate (Song et al. 1999) and therefore could not be used to examine foregut development. ActRIIA ${ }^{+/-}$, ActRIIA $^{-/-}$, and ActRIIB ${ }^{+/-}$embryos displayed no detectable malformations of the spleen (Oh and Li 1997; Song et al. 1999), or the stomach, pancreas, and liver (Fig. 1A).

ActRIIB $^{-/-}$and ActRIIA ${ }^{+/-} \mathrm{B}^{-/-}$mutants showed multiple foregut patterning defects, including an anteriorly directed transformation of the posterior stomach. The mammalian stomach is patterned along its AP axis (Fig. 1G; Rubio 1992; Larsson et al. 1996). Normal posterior stomach contains mucin-negative vacuoles in columnar epithelium, adjacent to a thick mesenchymal layer (Fig. $1 \mathrm{~A}, \mathrm{G})$, whereas anterior stomach epithelium is normally squamous and nonvacuolated (Rubio 1992; Larsson et al. 1996). The spleen forms within posterior stomach mesenchyme (Apelqvist et al. 1997; Kim et al. 1997b). ActRIIB $^{-/-}$embryos had posterior stomachs comprised of poorly vacuolated, cuboidal epithelium adjacent to an 

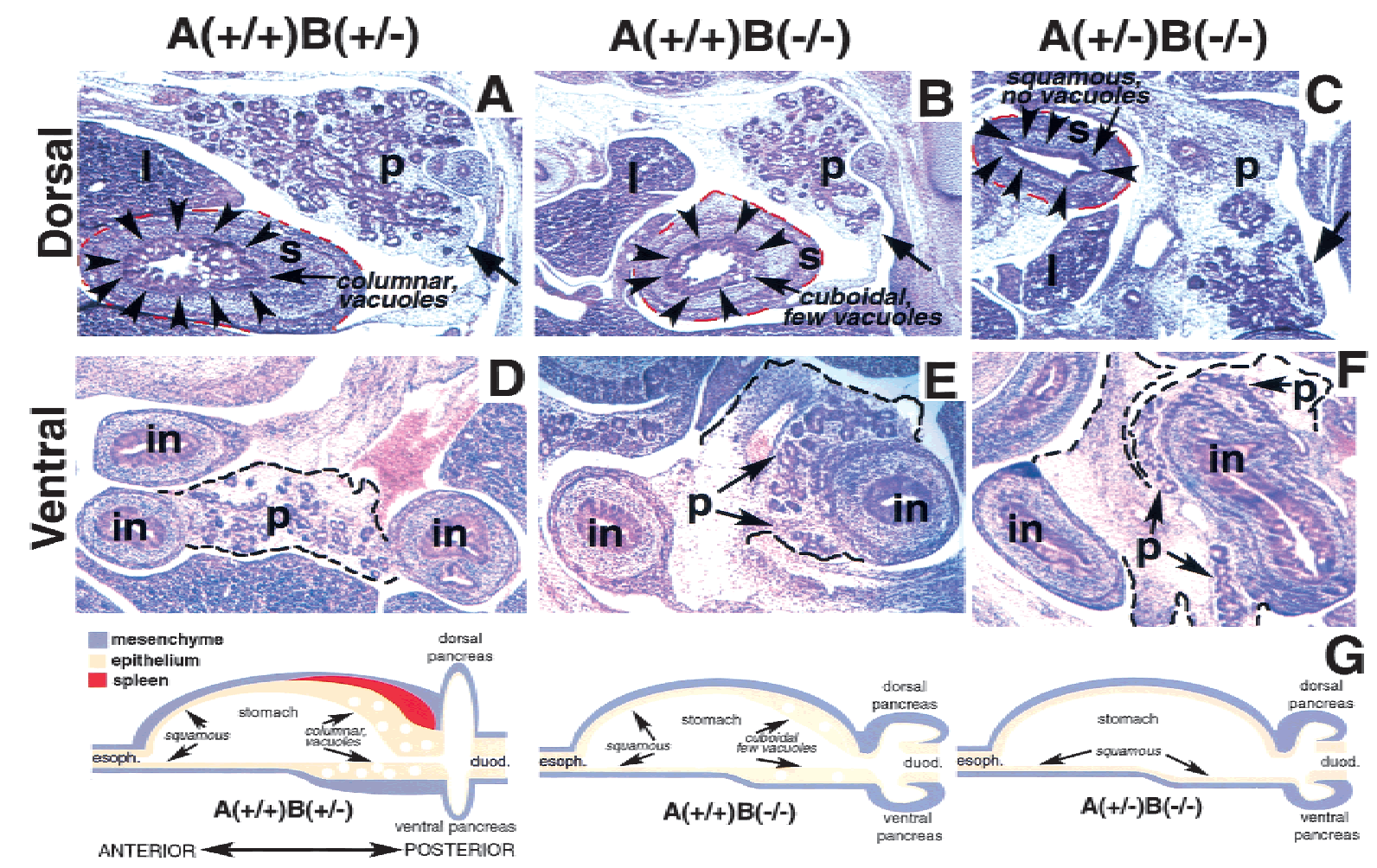

Figure 1. Pancreatic and stomach development in compound ActRIIA, ActRIIB mutant embryos at E13.5. In these and later panels, dorsal is toward the top and anterior toward the left. $(A-C)$ Dorsal pancreas (p), liver (1), and posterior stomach (s). (Arrowheads) Posterior stomach epithelium; (red dashes) stomach mesenchyme border; epithelial morphology (columnar, cuboidal, squamous) and number of vacuoles indicated. Stomach mesenchyme surrounds stomach epithelium. (Arrow) Pancreas epithelium. The anterior stomach and spleen are absent in these sections. (A) Sagittal section through ActRIIA ${ }^{+/+} \mathrm{B}^{+/-}$foregut. (B) Sagittal section through ActRIIB $^{-/-}$foregut. $(C)$ Sagittal section through ActRIIA ${ }^{+/-} \mathrm{B}^{-/-}$foregut. Note reduction of pancreatic epithelium in $B$ and $C$. In analysis of $>10$ embryos per genotype, we observe greater reductions of pancreatic size and epithelial components in ActRIIA ${ }^{+/-} \mathrm{B}^{-/-}$animals than in ActRIIA ${ }^{+/+} \mathrm{B}^{-/-}$animals. An embryo with a somewhat milder reduction of pancreatic epithelial structures is shown in $C$ to aid identification of the pancreas. $(D-F)$ Ventral pancreas $(\mathrm{p})$, ventral pancreas mesenchymal border (dashed line), ventral pancreas epithelium (arrows), and intestine (in). (D) Normal appearance of ventral pancreas. (E) ActRIIA ${ }^{+/+} \mathrm{B}^{-/-}$ventral pancreas surrounds and partly embeds in intestinal wall. $(F)$ ActRIIA ${ }^{+/-} \mathrm{B}^{-/-}$ventral pancreas surrounds and embeds within intestinal wall. (G) Summaries of sagittal foregut morphology in ActRIIA ${ }^{+/+} \mathrm{B}^{+/-}$, ActRIIA ${ }^{+/+} \mathrm{B}^{-/-}$, and ActRIIA ${ }^{+/-} \mathrm{B}^{-/-}$mutants. Esophagus (esoph.) and duodenum (duod.) are marked. Absent spleen, reduced pancreatic epithelium, and increased pancreatic mesenchyme in $\mathrm{ActRIIA}^{+/+} \mathrm{B}^{-/-}$and ActRIIA $^{+/-} \mathrm{B}^{-/-}$mutants are indicated.

attenuated mesenchymal layer (Fig. 1B,G). The pancreas in these mutants was reduced (Fig. 1B), and the spleen was absent, as shown previously (Oh and Li 1997). Thus, the stomach appeared anteriorized, and the posterior foregut organs like pancreas and spleen, were hypoplastic or absent in ActRIIB ${ }^{-/-}$mutants.

Stomach and pancreatic defects were most severe in ActRIIA $^{+/-} \mathrm{B}^{-/-}$embryos (Fig. 1C,G). The stomach in these mutants was comprised entirely of nonvacuolated, squamous epithelium, adjacent to a thin layer of mesenchyme: These mutants were also asplenic. Somatostatin, normally expressed in posterior, but not anterior stomach epithelium (Larsson et al. 1996; Rubio 1992), was reduced severely in the posterior stomach of ActRIIA $^{+/-} \mathrm{B}^{-/-}$embryos (J. Lee, E. Harmon, S. Kim, unpubl.). At E13.5 (Fig. 1C) and later stages (see below), the pancreas in these animals was reduced drastically. Wildtype ventral pancreas attaches to only one side of adjacent intestines (Fig. 1D). In contrast, the ventral pancreas in ActRIIA ${ }^{+/+} \mathrm{B}^{-/-}$and ActRIIA ${ }^{+/-} \mathrm{B}^{-/-}$embryos was abnormally merged with adjacent intestines, forming a partially encircling annulus (Fig. 1E-G). Thus, type II activin receptors are required both for morphogenesis within foregut organs, and for establishment of boundaries between foregut organs.

Shh and the LIM homeodomain transcription factor Islet1 (Isl1) are both regulators and molecular markers of foregut axial specification (Ahlgren et al. 1997; Apelqvist et al. 1997; Bellusci et al. 1997; Kim et al. 1997a; Hebrok et al. 1998; Litingtung et al. 1998). In a previous analysis of chick pancreatic development in vitro, we showed that activin signals from notochord may repress Shh expression in embryonic pancreas endoderm (Hebrok et al. 1998). Early in wild-type mouse foregut development, $\mathrm{Shh}$ is expressed in anterior stomach epithelium (Fig. 2A,I), but absent from posterior stomach (Fig. 2A,C,I). Ectopic expression of $S h h$ in posterior foregut epithelium results in asplenia, disrupted pancreatic morphogenesis, 
Figure 2. Posteriorly shifted gene expression along the AP foregut axis in ActRIIA ${ }^{+/-} \mathrm{B}^{-/}$mutants. $(A-H)$ Anterior stomach (as), posterior stomach (ps). $(A-D)$ Shh expression in stomach epithelium detected by in situ hybridization in sagittal sections of E11.5 and E13.5 embryos. (A) Shh expressed in epithelium of wild-type anterior stomach but not in posterior stomach. (Dashed lines) Combined boundaries of dorsal pancreas and spleen anlagen. Pancreatic epithelium is absent from this tissue section. $(B)$ Expanded Shh expression domain in E11.5 ActRIIA ${ }^{+/-} \mathrm{B}^{-/-}$embryos includes the posterior stomach epithelium. Dorsal pancreas and splenic anlagen (dashed lines) are reduced. $(C-D)$ Anterior stomach is not present in the section plane. Posterior stomach epithelium outlined by dashed lines. (Arrowhead) Pancreas. (C) Shh not detected in E13.5 wild-type posterior stomach epithelium. (D) Ectopic Shh expression in E13.5 ActRIIA $^{+/-} \mathrm{B}^{-/-}$posterior stomach epithelium. $(E-H)$ Isl1 in pancreatic and stomach mesenchyme at stage E11.5 and E13.5. Is11-expressing nuclei are stained brown, blue nuclear counterstain is Gill's hematoxylin. Stomach epithelium, which does not express Is11, marked by dashed lines in E and F. (E) Isl1 expression in wild-type dorsal pancreatic (panc.) mesenchymal cells (arrowhead) and mesenchyme surrounding stomach epithelium. (Arrows) Anterior boundary of Isl1 expression in foregut mesenchyme. $(F)$ Reduced Is11 expression in dorsal pancreas (panc.) mesenchyme (star), and in stomach mesenchyme. Isl1 expression detected only in posterior stomach mesenchyme. Note reduced dorsal pancreas size in the ActRIIA ${ }^{+/-} \mathrm{B}^{-/-}$mutant. $(G)$ Isl1 expression in E13.5 wild-type pancreas. $(H)$ Reduced pancreatic IsI1 expression in E13.5 $\mathrm{A}^{+/-} \mathrm{B}^{-/-}$mutants. Over 30,000 nuclei were scored in E13.5 wild-type and $\mathrm{A}^{+/-} \mathrm{B}^{-/-}$embryos. Twenty-six Isl1-positive nuclei were counted in $\mathrm{A}^{+/-} \mathrm{B}^{-/-}$animals vs. 2845 in wild type. (I) Summary of gene expression observed in wild-type and ActRIIA ${ }^{+/-} \mathrm{B}^{-/-}$mutant foregut.

and pancreatic cell differentiation within intestinal wall (Apelqvist et al. 1997). In ActRIIA ${ }^{+/-} \mathrm{B}^{-/-}$embryos, Shh was expressed inappropriately in both anterior (Fig. 2B,I) and posterior stomach epithelium (Fig. 2B,D,I); adjacent pancreatic and splenic primordia were reduced. These results and those of Apelqvist et al. (1997) suggest that the observed foregut phenotypes in ActRIIA ${ }^{+/-} \mathrm{B}^{-/-}$mutants result from $S h h$ misexpression. Thus, activin receptor-mediated signaling restricts $S h h$ expression in mouse foregut endoderm.

Pancreatic islet cells and dorsal mesenchyme fail to develop in mice lacking Isl1 (Ahlgren et al. 1997). At E11.5, Is11 expression was detected in wild-type anterior and posterior stomach mesenchyme, pancreatic mesenchyme (Fig. 2E,I), and pancreatic epithelium (Ahlgren et al. 1997; Kim et al. 1997b). By E13.5, Is11 expression in normal foregut was detected only in pancreatic epithelial cell clusters (Fig. 2G). In contrast, ActRIIA ${ }^{+/-} \mathrm{B}^{-/-}$em-

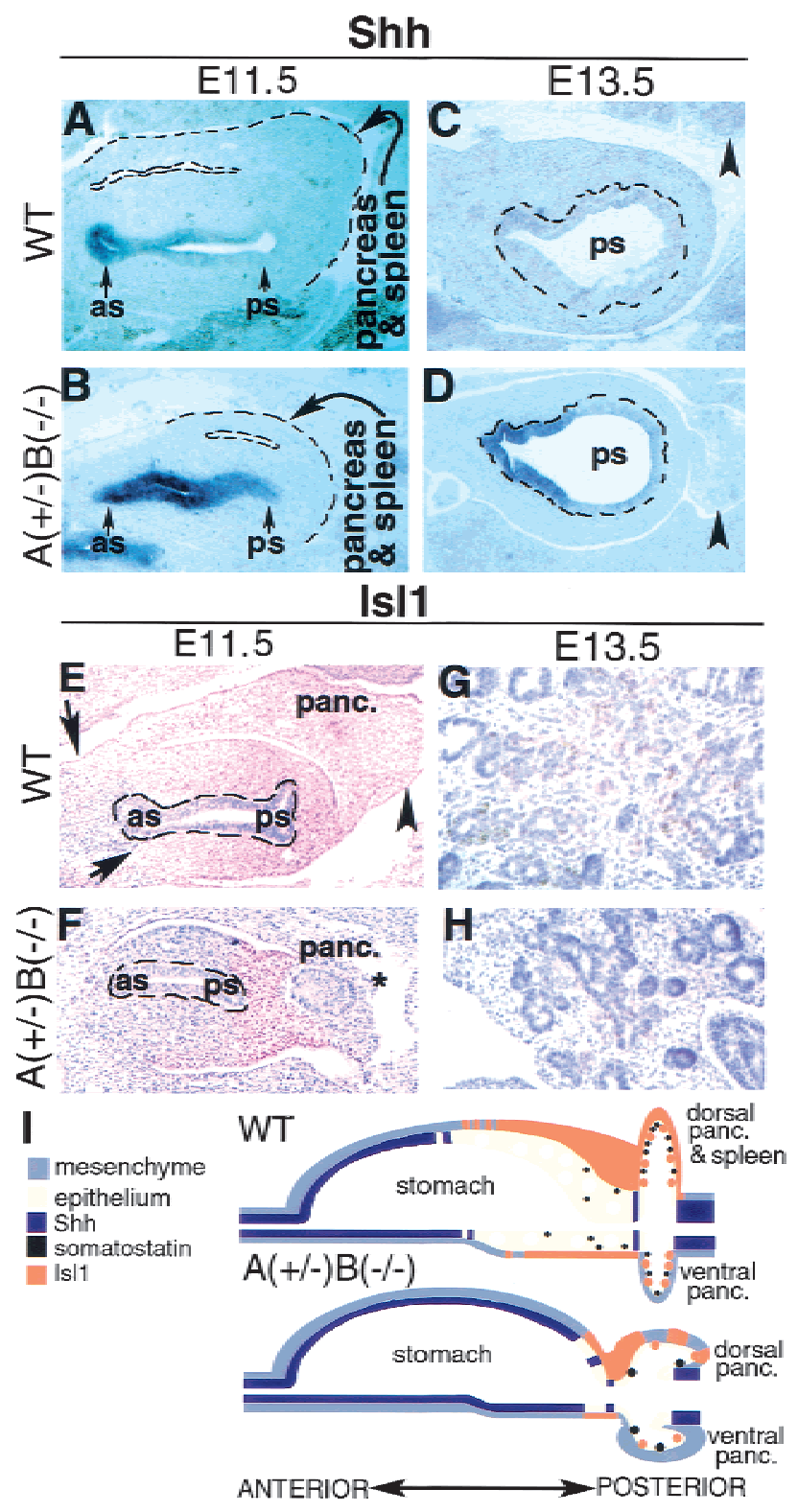

bryos had decreased Is11 expression. At E11.5 Is11 expression in the stomach was restricted to posterior mesenchyme, and was reduced in pancreatic mesenchyme (Fig. 2F,II. By E13.5, pancreatic epithelial Is11 expression in ActRIIA $^{+/-} \mathrm{B}^{-/-}$mutants was reduced severely (Fig. $2 \mathrm{H}$ ) compared to wild type. Thus, type II activin receptormediated signaling regulates Isl1 expression in foregut mesenchyme and pancreas epithelium.

Altered expression of pancreatic Isl1 in animals with ActRIIA and ActRIIB mutations prompted studies of pancreatic cell differentiation. Analysis at stage E13.5 revealed a severe reduction of insulin and glucagon expression, but only slight reduction of carboxypeptidase A expression in ActRIIA ${ }^{+/-} \mathrm{B}^{-/-}$embryos (Fig. 3A). This pancreatic endocrine defect persisted throughout gestation (Fig 3B). Pancreatic islets form by E17.5-18.5, and at this stage in ActRIIB ${ }^{-/-}$and ActRIIA ${ }^{+/-} \mathrm{B}^{-/-}$embryos we observed a marked reduction in the size and number of 

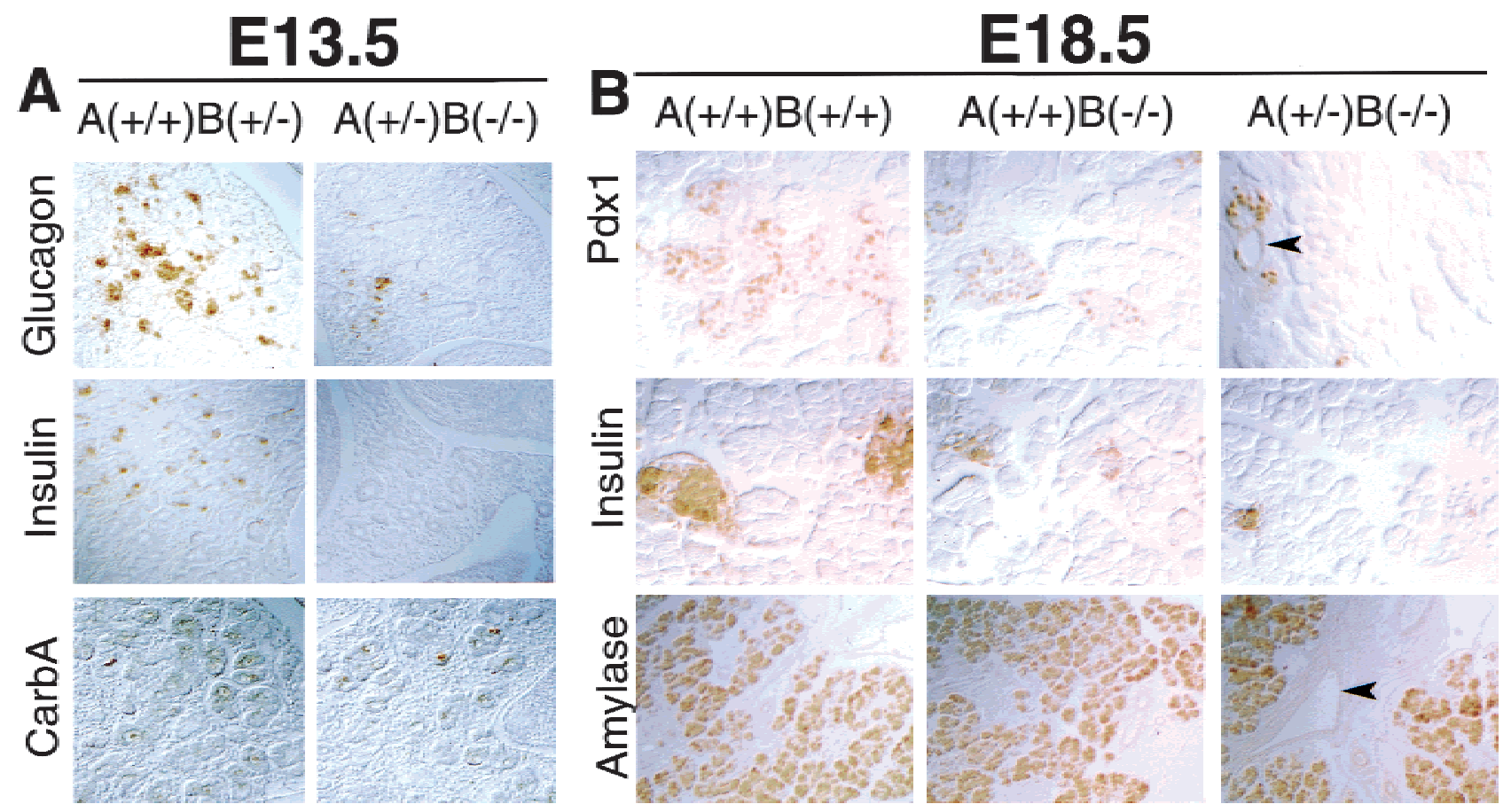

Figure 3. Defective endocrine development in type II activin receptor mutants. (A) Stage E13.5. Immunohistochemistry reveals a severe reduction of glucagon and insulin expression in ActRIIA ${ }^{+/-} \mathrm{B}^{-/-}$embryos. Little change in carboxypeptidase A (carbA) expression is observed. Data shown for dorsal pancreas, but the same result is also observed in the ventral pancreas. $(B)$ Stage E18.5. Reduction in $P d x 1$ and insulin-expressing cells in embryos with ActRII mutations. $P d x 1$-expressing cells in ActRIIA ${ }^{+/-} \mathrm{B}^{-/-}$embryos form small clusters contiguous with ductules (arrowhead). Increased ductular cell expression of $P d x 1$ is also noted. Amylase expression is well maintained. An increase of ductal structures in fibrotic mesenchyme (arrowhead) is evident in the ActRIIA ${ }^{+/-} \mathrm{B}^{-/-}$pancreas stained for amylase expression. Magnification: $40 \times$ in $A ; 100 \times$ in amylase panels in $B ; 200 \times$ in remaining panels in $B$.

islets expressing the transcription factor $P d \times 1$ and insulin (Fig. 3B). E18.5 ActRIIA ${ }^{+/-} \mathrm{B}^{-/-}$embryos have no detectable somatostatin in islets, and in the posterior stomach (data not shown). In contrast, levels of amylase expression within intact portions of pancreas in these mutants appeared unchanged (Fig. 3B). Thus, type II activin receptors are required for endocrine differentiation in pancreas and stomach.

Inactivation of ActRIIA and ActRIIB in adult mice resulted in pancreatic islet hypoplasia (Fig. 4A,B). Mice with three or four null alleles of type II activin receptors were not viable; however, ActRIIA ${ }^{-/-}$, ActRIIB $^{-/-}$, and ActRIIA $^{+/-} \mathrm{B}^{+/-}$animals were viable and fertile (Matzuk et al. 1995; Oh and Li 1997; Song et al. 1999). Histologic and morphometric analysis of pancreatic islets in ActRIIB $^{-1-}$ and ActRIIA ${ }^{+/-} \mathrm{B}^{+/-}$animals revealed a significant reduction of islet size in these animals (Fig. 4B). Average islet size in wild-type animals is $29,800 \pm 4600$ $\mu \mathrm{m}^{2}$, in ActRIIB ${ }^{-/-}$animals is $9300 \pm 790 \mu \mathrm{m}^{2}$, and in ActRIIA $^{+/-} \mathrm{B}^{+/-}$animals is $5600 \pm 620 \mathrm{\mu m}^{2}$. Islet size in ActRIIA $^{-/-}$mice was not reduced significantly (J. Lee and S. Kim, unpubl.).

Characterization of $>100$ ActRIIA ${ }^{+/-} \mathrm{B}^{+/-}$mutants reveals that these animals do not have detectable axial defects, or severely disrupted Shh expression (Song et al. 1999; S.P. Oh and S. Kim, unpubl.). It is therefore unlikely that changes in islet size and function in ActRIIA $^{+/-} \mathrm{B}^{+/-}$animals are secondary to axial patterning defects. Thus, independent of their roles in axial patterning, type II activin receptors also regulate pancreatic islet development.

Pancreatic expression of a dominant-negative type II activin receptor in transgenic mice results in islet hypoplasia (Yamaoka et al. 1998; Shiozaki et al. 1999). Transgenic animals had hypoinsulinemia and impaired glucose tolerance in one study (Yamaoka et al. 1998), but had normal serum insulin levels and glucose tolerance in a more recent study (Shiozaki et al. 1999). Thus, these previous studies have not established a role for activin signaling in pancreatic endocrine function. Our studies revealed impaired glucose tolerance in $\mathrm{ActRIIB}^{-/-}$and ActRIIA $^{+/-} \mathrm{B}^{+/-}$mutants (Fig. $4 \mathrm{C}$ ). In glucose tolerance tests after overnight fasting, ActRIIB ${ }^{-/-}$animals had abnormally elevated peak blood glucose levels, but otherwise normal glucose handling. In contrast, ActRIIA ${ }^{+/-} \mathrm{B}^{+/-}$ animals had abnormal peak glucose levels, and a prolonged ensuing hyperglycemia (Fig. 4C). ActRIIA ${ }^{-/-}$mice had normal-sized islets and showed no impairment of glucose tolerance $(n=9 ;$ H. Schrewe, unpubl.). Thus, the severity of impaired glucose tolerance depended on the type and degree of activin receptor inactivation and correlated with the degree of islet hypoplasia (Fig. 4B). We then measured insulin levels in wild-type and ActRIIA $^{+/-} \mathrm{B}^{+/-}$animals after overnight fasting and glucose challenge. Insulin levels after overnight fasting were similar in wild-type and ActRIIA ${ }^{+/-} \mathrm{B}^{+/-}$animals. 
Figure 4. Pancreas morphology and glucose tolerance in adult ActRII mutants. $(A, B)$ Islet hypoplasia in ActRIIB $^{-/-}$mutants and ActRIIA ${ }^{+/-} \mathrm{B}^{+/-}$compound heterozygotes. (A) (Top row) Tissues stained by hematoxylin and eosin (H\&E). Islets (arrowheads) from wild-type, $\mathrm{B}^{-/-}$and $\mathrm{A}^{+/-} \mathrm{B}^{+/-}$adults. (Lower row) Insulin expression in wild-type and mutant islets detected in central islet cells, indicating preserved islet architecture. Expression of glucagon, PP and somatostatin by peripheral islet cells surrounding centrally located insulin ${ }^{+}$cells is preserved in mutant islets (data not shown). (B) Morphometric analysis of islet area in pancreata from wild-type and mutant adults. For wild-type, $n=259 ;$ ActRIIB $^{-/-}, n=259$; ActRIIA $^{+/-} \mathrm{B}^{+/-}, n=237$. Distribution of islet areas $<5000 \mu \mathrm{m}^{2}, 5000-10,000 \mu \mathrm{m}^{2}$, or $>10,000 \mu^{2}$ is shown as a percentage of islets measured. $\mathrm{P}<0.001$ for wild type vs. ActRIIA $^{+/-} \mathrm{B}^{+/-}$and $\mathrm{P}<0.005$ for wild type vs. ActRIIB ${ }^{-/-}$. Data presented as average \pm standard error of the mean (s.E.M.). (C) Glucose tolerance testing of $12-16$ week-old adult male mice after $14 \mathrm{hr}$. overnight fast. Wild type, $n=6$; ActRIIB $^{-/-}, n=5$; ActRIIA $^{+/-} \mathrm{B}^{+/-}, n=5$. Identical testing of ActRIIA $^{-/-}$animals $(n=9)$ revealed no significant difference in glucose tolerance compared to wild-type controls (H. Schrewe, unpubl.). (D) Serum insulin levels in wild-type and ActRIIA $^{+/-} \mathrm{B}^{+/-}$animals after overnight fasting and $30 \mathrm{~min}$ after glucose challenge. Data presented as average \pm S.E.M.

Insulin levels were reduced inappropriately, however, in ActRIIA $^{+/-} \mathrm{B}^{+/-}$animals after glucose challenge (Fig. 4D), suggesting that the observed impairment in glucose tolerance may result from inadequate insulin production or secretion. Thus, while not overtly diabetic, trans-heterozygous type II activin receptor mutants provide a polygenic animal model of impaired glucose tolerance.

Activin and Hedgehog signal transduction pathways are relatively well-characterized, and provide attractive molecular targets for modifying growth of vital tissues like pancreatic islets. Biochemical and genetic data suggest that ActRIIA and ActRIIB can interact with a number of ligands, including activins A and B, Vg-1, Nodal, BMP4, BMP7, Gdf11, and Lefty2. Results from this study and others (Attisano et al. 1993; Oh and Li 1997; McPherron et al. 1999; Song et al. 1999) suggest that multiple ligands may pattern foregut development through type II activin receptor pathways. Type II activin receptors also have known interactions with type I receptors (for review, see Heldin et al. 1997), and intracellular signal transduction proteins like Smad2 and Smad3 (for review, see Massagué and Chen 2000; Wrana 2000). Thus, TGF- $\beta$ signaling pathways may govern both cell autonomous and nonautonomous processes in foregut development. Understanding the role of activin signaling in embryonic and adult organogenesis may aid development of novel diagnostic and cell-replacement strategies for diseases like diabetes mellitus.

\section{Materials and methods}

Generation and genotyping of ActRIIA and ActRIIB mutant mice Targeted disruption of ActRIIA (Song et al. 1999) and ActRIIB (Oh and Li 1997) has been described. Both ActRIIA and ActRIIB heterozygotes were intercrossed to generate embryos and adult mice of the desired genotype. The genotypes of all offspring were determined by Southern blot or PCR analysis of genomic DNA as described previously (Oh and Li 1997; Song et al. 1999). All mice were on a mixed 129/Sv-C57BL/6J hybrid genetic background.

Histologic and morphometric methods

Pancreas was fixed by immersion in $10 \%$ neutral buffered formalin, dehydrated, embedded, sectioned $(6 \mu \mathrm{m})$, and stained with hematoxylin and eosin (Kim et al. 1997a). Each section was subjected to morphometry using the Metamorph Image analyzer (Universal Imaging Corp., West Chester, PA). The area of each pancreatic islet was measured and the data was subjected to statistical analysis using Excel (Microsoft). ActRIIA and ActRIIB in situ hybridization probes are available on request from Dr. H. Schrewe. In situ hybridizations were performed as described in Kim et al. (1997a). Is11 antibody was obtained from the Developmental Hybridoma bank (University of Iowa), was used at 1:100 dilution, and detected in paraffin-embedded tissue sections thrice microwaved in antigen unmasking solution (Vector, Burlingame, CA) for $5 \mathrm{~min}$. Antibodies against $P d \times 1$ were a gift from Dr. C. Wright (Vanderbilt University) and used as described in O'Reilly et al. (1997) and Wu et al. (1997). Other primary antibodies including anti-insulin, anti-glucagon, anti-carboxypeptidase A, and anti-amylase were used as described previously (Kim et al. 1997a; Hebrok et al. 1998, 2000).

Glucose-tolerance testing and insulin measurements

Glucose tolerance and insulin levels in male mice were measured as described by Bruning et al. (1998), except that glucose levels were measured with a Glucometer Elite (Bayer, Inc.). 


\section{Acknowledgments}

We thank Q. Wu and L. Chen for expert assistance; D. Ko for assistance with morphometry; and Drs. M.P. Scott, W. Talbot, G. Barsh, D. Kaiser, J. Shiziru, and members of the Kim lab for helpful comments on the manuscript. M.H. was supported by a Howard Hughes Medical Institute (HHMI) postdoctoral fellowship. D.A.M. is an investigator in the HHMI. S.K.K. was supported by a HHMI Physician Postdoctoral Fellowship, a Pew Biomedical Scholars award, the Donald E. and Delia B. Baxter Foundation, and a career development award from the American Diabetes Association.

The publication costs of this article were defrayed in part by payment of page charges. This article must therefore be hereby marked "advertisement" in accordance with 18 USC section 1734 solely to indicate this fact.

\section{References}

Ahlgren, U., Pfaff, S., Jessell, T.M., Edlund, T., and Edlund, H. 1997. Independent requirement for ISL1 in the formation of pancreatic mesenchyme and islet cells. Nature 385: 257-260.

Apelqvist, A., Ahlgren, U., and Edlund, H. 1997. Sonic hedgehog directs specialised mesoderm differentiation in the intestine and pancreas. Curr. Biol. 7: 801-804.

Attisano, L., Carcamo, J., Ventura, F., Weis, F.M., Massague, J., and Wrana, J.L. 1993. Identification of human activin and TGF beta type I receptors that form heteromeric kinase complexes with type II receptors. Cell 75: 671-680.

Bellusci, S., Furuta, Y., Rush, M.G., Henderson, R., Winnier, G., and Hogan, B.L.M. 1997. Involvement of Sonic hedgehog (Shh) in mouse embryonic lung growth and morphogenesis. Development 124: 5363.

Bruning, J.C., Winnay, J., Bonner-Weir, W., Taylor, S.I., Accili, D., and Kahn, C.R. 1997. Development of a novel polygenic model of NIDDM in mice heterozygous for IR and IRS-1 null alleles. Cell 88: $561-572$.

Feijen, A., Goumans, J.M., and van den Eijnden-van Raaij, A.J.M. 1994. Expression of activin subunits, activin receptors and follistatin in postimplantation mouse embryos suggests specific developmental functions for different activins. Development 120: 3621-3637.

Hebrok, M., Kim, S.K., and Melton, D.A. 1998. Notochord repression of endodermal Sonic hedgehog permits pancreas development. Genes \& Dev. 12: 1705-1713.

Hebrok, M., Kim, S.K., St.-Jacques, B., McMahon, A.P., and Melton, D.A. 2000. Regulation of pancreas development by Hedgehog signaling. Development (in press).

Heldin, C.H., Miyazono, K., and ten Dijke, P. 1997. TGF- $\beta$ signalling from cell membrane to nucleus through SMAD proteins. Nature 390: 465-471.

Hoodless, P.A., Tsukazaki, T., Nishimatsu, S., Attisano, L., Wrana, J.L., and Thomsen, G.H. 1999. Dominant-negative Smad2 mutants in hibit activin/Vg1 signaling and disrupt axis formation in Xenopus. Dev. Biol. 207: 364-379.

Kim, S.K. and Melton, D.A. 1998. Pancreas development is promoted by cyclopamine, a Hedgehog signaling inhibitor. Proc. Natl. Acad. Sci. 95: 13036-13041.

Kim, S.K., Hebrok, M., and Melton, D.A. 1997a. Notochord to endoderm signaling is required for pancreas development. Development 124: 4243-4252.

. 1997b. Pancreas development in the chick embryo. Cold Spring Harbor Symp. Quant. Biol. 62: 377-383.

Larsson, L.I., Madsen, O.D., Serup, P., Jonsson, J., and Edlund, H. 1996. Pancreatic-duodenal homeobox 1 -role in gastric endocrine patterning. Mech. Dev. 60: 175-184.

Levin, M., Johnson, R.L., Stern, C.D., Kuehn, M., and Tabin, C.J. 1995. A molecular pathway determining left-right asymmetry in chick embryogenesis. Cell 82: 803-814.

Litingtung, Y., Lei, L., Westphal, H., and Chiang, C. 1998. Sonic hedgehog is essential to foregut development. Nat. Genet. 20: 58-61.

Manova, K., De Leon, V., Angeles, M., Kalantry, S., Giarre, M., Attisano, L., Wrana, J., and Bachvarova, R.F. 1995. mRNAs for activin receptors II and IIB are expressed in mouse oocytes and in the epiblast of pregastrula and gastrula stage mouse embryos. Mech. Dev. 49: 3-11.

Massagué, J. and Chen, Y.-G. 2000. Controlling TGF- $\beta$ signaling. Genes
\& Dev. 14: 627-644.

Matzuk, M.M., Kumar, T.R., and Bradley, A. 1995. Different phenotypes for mice deficient in either activins or activin receptor type II. Nature 374: 356-360.

McPherron, A.C., Lawler, A.M., and Lee, S.-J. 1999. Regulation of anterior/posterior patterning of the axial skeleton by growth/differentiation factor 11. Nat. Genet. 22: 260-264.

Oh, S.P. and Li, E. 1997. The signaling pathway mediated by the type IIB activin receptor controls axial patterning and lateral asymmetry in the mouse. Genes \& Dev. 11: 1812-1826.

O'Reilly, L.A., Gu, D., Sarvetnick, N., Edlund, H., Phillips, J.M., Fulford, T., and Cooke, A. 1997. $\alpha$-Cell neogenesis in an animal model of IDDM. Diabetes 46: 599-606.

Pepicelli, C.V., Lewis, P.M., and McMahon, A.P. 1998. Sonic hedgehog regulates branching morphogenesis in the mammalian lung. Curr. Biol. 8: 1083-1086.

Roberts, D.J., Johnson, R.L., Burke, A.C., Nelson, C.E., Morgan, B.A., and Tabin, C. 1995. Sonic hedgehog is an endodermal signal inducing BMP-4 and Hox genes during induction and regionalization of the chick hindgut. Development 121: 3163-3174.

Rubio, C.A. 1992. Five types of pyloric cells in the antral mucosa of the stomach. Pathol. Res. Pract. 188: 157-161.

Sasai, Y. and De Robertis, E.M. 1997. Ectodermal patterning in vertebrate embryos. Dev. Biol. 182: 5-20.

Shiozaki, S., Tajima, T., Zhang, Y.Q., Furukawa, M., Nakazato, Y., and Kojima, I. 1999. Impaired differentiation of endocrine and exocrine cells of the pancreas in transgenic mouse expressing the truncated type II activin receptor. Biochim. Biophys. Acta 1450: 1-11.

Song, J., Oh, S.P., Schrewe, H., Nomura, M., Lei, H., Okano, M., Gridley, T., and Li, E. 1999. The type II activin receptors are essential for egg cylinder growth, gastrulation, and rostral head development in mice. Dev. Biol. 213: 157-169.

Verschueren, A., Dewulf, N., Goumans, M.J., Lonnoy, O., Feijen, A., Grimsby, S., Vandi Spiegle, K., ten Dijke, P., Moren, A., Vanscheeuwijck, P. et al. 1995. Expression of type I and type IB receptors for activin in midgestation mouse embryos suggests distinct functions in organogenesis. Mech. Dev. 52: 109-123.

Wrana, J.L. 2000. Regulation of Smad activity. Cell 100: 189-192.

Wu, K.L., Gannon, M., Peshavaria, M., Offield, M.F., Henderson, E., Ray, M., Marks, A., Gamer, L.W., Wright, C.V., and Stein, R. 1997. Hepatocyte nuclear factor $3 \beta$ is involved in pancreatic beta-cell- specific transcription of the pdx-1 gene. Mol. Cell. Biol. 10: 6002-6013.

Yamaoka, T., Idehara, C., Yano, M., Matsushita, T., Yamada, T., Li, S., Moritani, M., Hata, J., Sugino, H., Noji, S. et al. 1998. Hypoplasia of pancreatic islets in transgenic mice expressing activin receptor mutants. J. Clin. Invest. 102: 294-301. 


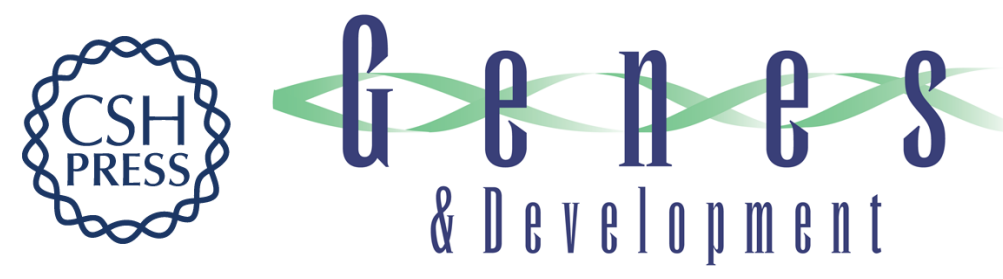

\section{Activin receptor patterning of foregut organogenesis}

Seung K. Kim, Matthias Hebrok, En Li, et al.

Genes Dev. 2000, 14:

Access the most recent version at doi:10.1101/gad.14.15.1866

References This article cites 31 articles, 10 of which can be accessed free at: http://genesdev.cshlp.org/content/14/15/1866.full.html\#ref-list-1

License

Email Alerting Receive free email alerts when new articles cite this article - sign up in the box at the top Service right corner of the article or click here.

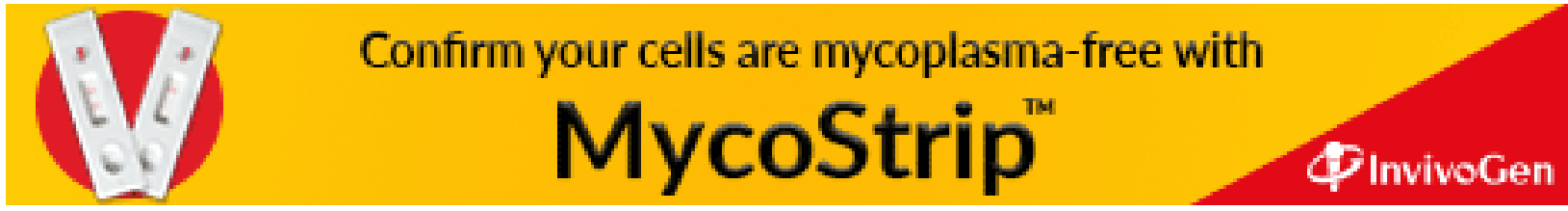

